\title{
Efficient and Secure Decentralized Network Size Estimation
}

\author{
Nathan Evans ${ }^{1}$, Bartlomiej Polot ${ }^{2}$, and Christian Grothoff ${ }^{2}$ \\ 1 Symantec Research Labs, \\ nathan_evans@symantec.com \\ 2 Free Secure Network Systems Group, \\ Network Architectures and Services, \\ Technische Universität München, \\ \{bart,grothoff\}@net.in.tum.de
}

\begin{abstract}
The size of a Peer-to-Peer (P2P) network is an important parameter for performance tuning of $\mathrm{P} 2 \mathrm{P}$ routing algorithms. This paper introduces and evaluates a new efficient method for participants in an unstructured P2P network to establish the size of the overall network. The presented method is highly efficient, propagating information about the current size of the network to all participants using $O(|E|)$ operations where $|E|$ is the number of edges in the network. Afterwards, all nodes have the same network size estimate, which can be made arbitrarily accurate by averaging results from multiple rounds of the protocol. Security measures are included which make it prohibitively expensive for a typical active participating adversary to significantly manipulate the estimates. This paper includes experimental results that demonstrate the viability, efficiency and accuracy of the protocol.
\end{abstract}

Keywords: Peer-to-Peer, protocol design, network security.

\section{Introduction}

Individual peers participating in unstructured networks, such as Peer-to-Peer (P2P) networks, ad-hoc wireless networks and sensor networks, can benefit from knowing the size (total number of participants) of the network. Peers in unstructured P2P networks which know the network size can make intelligent decisions with respect to content replication, message routing and forwarding and the overall cost of operations. Additionally, nodes in a sensor network can use such data to gauge the health of the overall network, calculate on/off time to save energy, selectively route messages, and generate alerts.

This paper describes the design, implementation and experimental results of a protocol that provides all peers in a structured or unstructured P2P network with an accurate estimate of the total number of peers in the network. The primary motivation for our work are the requirements of various $\mathrm{P} 2 \mathrm{P}$ routing protocols 4612; these protocols explicitly require a network size estimate to tune parameters responsible for routing, path selection and content replication. 
The focus of our design is to provide security in the context of an open and completely decentralized network architecture. While it would be possible to strengthen the security of our design with trusted centralized services - for example by preventing a Sybil-attack with a centralized registration requirement - our design does not require a centralized authority and is intended to provide security in the presence of actively participating adversaries. A key difference to existing proposals is that in our design there are no peers with special roles in the process; this eliminates the possibility of malicious peers abusing such roles.

A central goal of our design — which is generally not satisfied by many other network size estimation algorithms [11114 — is that all peers are supposed to participate in calculating a network size estimate at roughly the same time and obtain the same result. This is achieved by a controlled flood of the network with the size estimation information, costing $O(|E|)$ messages per round. In practice, the constant factor is typically between one and two; in other words, the algorithm can be expected to only generate $|E|$ messages per round.

The basic idea behind our algorithm is to flood the network with the identity of the peer whose identity is closest to a particular key $T$. Each peer's identity is generated when the peer starts the first time. The key $T$ is not chosen by any peer but instead is generated from the start time $S$ of the current round. Despite using time, we specifically do not assume that the clocks in the network are closely synchronized; our protocol ensures that in the worst case individual peers with significant clock skew only cause a bounded amount of additional network traffic.

Our protocol considers many other important networking issues as well. The protocol is very efficient as it requires only $O(1)$ state per peer and does not require peers to establish new connections (we assume the network graph is connected). The amount of work required by each node is based only on the number of edges of the node, so the load between peers is typically balanced. Given that our protocol floods the network with size estimation information, peers randomly delay messages to avoid spikes in network traffic. Finally, our design handles network churn well, and allows the system designer to trade-off computational efficiency for security and bandwidth for accuracy.

The remainder of the paper is structured as follows. In Section 2, we present related work on methods for network size estimation for P2P networks. Section 3 then presents our protocol. In Section 4 we analyze the security aspects of the protocol. Experimental results obtained using large-scale emulation are given in Section 5.

\section{Related Work}

Algorithms for estimating the size of a P2P network can be categorized into algorithms for structured overlays, which typically exploit statistical properties of an existing routing table from a DHT, and algorithms for unstructured overlays, which make no assumptions about the structure of the underlying network. 


\subsection{Network Size Estimation for Structured Overlays}

Structured overlays construct routing tables at each peer according to particular rules that enable efficient routing of messages to the peer with the "closest" identifier with respect to a given key 1518. In these structured overlays, the distance to the nearest neighbors in the routing table can be used as a first network size estimate as it correlates with the network size [17.

As node identifiers are often not perfectly uniform, searching the structured overlay for the closest node to various randomly selected keys can be used to get accurate network size estimates [17. Given a DHT routing algorithm with a typical cost of $O(\log n)$, network size estimation for all nodes using this method would be $O(n \log n)$. When compared to the method presented in this paper, a key disadvantage of existing methods for structured networks is that they rely on the security of the underlying routing algorithm; actively participating malicious nodes have thus the potential to significantly skew the network size estimate. Furthermore, for any of the structured methods that we are aware of, different nodes will virtually always compute somewhat different network size estimates.

\subsection{Network Size Estimation for Unstructured Overlays}

Several algorithms for unstructured overlays are based on sampling. Examples include Sample \& Collide, Random Tour and Hops Sampling. In Sample \& Collide 14, each peer starts bounded random walks to sample random peers in the network and uses the collision information and the birthday paradox to estimate the size of the network. In Random Tours [14], a message tours the network until it reaches the initiator; the size estimate is then computed based on a counter in the message that was incremented by each peer on the tour. Hops Sampling [1] works by flooding the network with a message containing a hop count. Peers report back to the initiator with a probability inverse to the hop count they received. The network size estimate is then the sum over all distances of the number of replies received for a particular distance divided by the reply-probability for that distance.

As described, these methods generate results for just one peer in the network, resulting in a high amount of bandwidth used overall (assuming each peer requires an estimate). Also, different peers will have potentially significantly different network size estimates. While these approaches do not assume a particular network structure for routing, they do still make implicit assumptions about the structure of the overlay topology and may significantly underestimate the network size if the overlay topology happens to have a structure that is unfavorable to the algorithm. For example, a circular topology would result in a network size estimate of $n^{2}$ for Sample \& Collide.

Other algorithms, such as Gossip-based Aggregation [8], achieve a somewhat more uniform estimate for all participating nodes at the cost of sensitivity to node failures. Gossip-based Aggregation starts with one peer setting a local state to 1 while all other peers set their local state to 0 . Peers continuously connect to randomly selected peers, and exchange states in pairs. Each peer then replaces 
its state with the average of both values. After a predefined number of iterations, all peers are supposed to end up with a value close to $1 / n$ where $n$ is the size of the network. A method that addresses the problem of who should set the state to 1 has been proposed [20], but only works in certain structured networks and retains other shortcomings of this approach, including high vulnerability to malicious peers.

A special case is the method proposed in [1] which attempts to produce a network size estimate using only "local" information. The idea behind this algorithm is to observe the number of new neighbors discovered in a breadth-first search of the network and estimate the network size based on the growth of this function. The authors claim to obtain accurate results with a breadth-first search of depth three, which makes this a "local" method. However, the way they constructed the topologies for their experiments does not seem to properly model the structure of actual networks. We were unable to reproduce their results on other network topologies.

The accuracy and performance of various size estimation methods for unstructured networks are compared in [16] using simulation. The authors identify the Sample \& Collide method as the strongest algorithm and state that it requires about 50 million messages in a random-graph topology of 100,000 nodes for an accuracy of $\pm 4 \%$. It should be noted that this is the overhead for an individual node to obtain an estimate; if each of the 100,000 nodes were to run the Sample \& Collide protocol, it would take 5 trillion messages to achieve this degree of accuracy.

None of these papers mention concrete implementations or discuss security concerns. Furthermore, all of them are clearly vulnerable to malicious participants. For example, in the case of sampling-based algorithms, malicious participants can manipulate walks that pass through them (allowing virtually unbounded manipulation of the network size estimates) or achieve a significant multiplier $(O(\sqrt{n}))$ to their network bandwidth in a denial-of-service attack by continuously initiating size estimation requests. Similarly, an active adversary can manipulate the exchanged values in gossip-based methods to change the size estimate in any direction.

\section{Our Approach}

We generate node identifiers by hashing the public key of the respective node. Node identifiers for benign nodes should therefore be statistically equivalent to random numbers from a uniform distribution. Furthermore, nodes are able to cryptographically sign messages using their respective private key.

Similar to the network size estimation algorithms for structured overlays, our network size estimation approach is based on the largest number of leading overlapping bits between any node identifier and a random key:

Theorem 1. Let $\bar{p}$ be the expected maximum number of leading overlapping bits between all $n$ random node identifiers in the network and a random key. Then the network size $n$ is approximately $2^{\bar{p}-0.332747}$. 
Proof. Let $X$ be the random variable for all $n$ identifiers and let $X_{i}$ be the number of overlapping bits for an individual random node identifier $i$.

The probability that a single random node identifier $i$ overlaps with at least $\alpha$ bits with a random key is

$$
P\left(X_{i} \geq \alpha\right)=2^{-\alpha}
$$

Then, the probability that a single random node identifier overlaps with less than $\alpha$ bits with a random key is

$$
P\left(X_{i}<\alpha\right)=1-2^{-\alpha} .
$$

The probability that the maximum number of leading overlapping bits for all $n$ random nodes is strictly less than $\alpha$ is

$$
P_{n}(X<\alpha):=: P\left(\bigwedge_{i} X_{i}<\alpha\right)=\left(P\left(X_{i}<\alpha\right)\right)^{n}=\left(1-2^{-\alpha}\right)^{n} .
$$

Then $E_{n}(X)$, the expected maximum number of leading overlapping bits between $n$ random node identifiers in the network is:

$$
\begin{aligned}
E_{n}(X) & :=: \sum_{\alpha=0}^{\infty} \alpha \cdot P_{n}(X=\alpha)=\sum_{\alpha=1}^{\infty} P_{n}(X \geq \alpha) \\
& =\sum_{\alpha=1}^{\infty}\left(1-P_{n}(X<\alpha)\right)=\sum_{\alpha=1}^{\infty}\left(1-\left(1-2^{-\alpha}\right)^{n}\right) \\
& =\sum_{\alpha=1}^{\log _{2} n}\left(1-\left(1-2^{-\alpha}\right)^{n}\right)+\sum_{\alpha=\log _{2} n+1}^{\infty}\left(1-\left(1-2^{-\alpha}\right)^{n}\right)
\end{aligned}
$$

Suppose $n$ is sufficiently large such that we can use $\lim _{n \rightarrow \infty}\left(1-\frac{x}{n}\right)^{n}=e^{-x}$. By substituting $\beta:=\alpha-\log _{2} n$ and $\gamma:=\log _{2} n-\alpha$ we then get:

$$
\begin{aligned}
E_{n}(X) & =\log _{2} n-\sum_{\gamma=0}^{\log _{2} n-1}\left(1-2^{\gamma-\log _{2} n}\right)^{n}+\sum_{\beta=1}^{\infty}\left(1-\left(1-2^{-\left(\beta+\log _{2} n\right)}\right)^{n}\right) \\
& =\log _{2} n-\sum_{\gamma=0}^{\log _{2} n-1}\left(1-\frac{2^{\gamma}}{n}\right)^{n}+\sum_{\beta=1}^{\infty}\left(1-\left(1-\frac{2^{-\beta}}{n}\right)^{n}\right) \\
& \approx \log _{2} n-\sum_{\gamma=0}^{\log _{2} n-1} e^{-2^{\gamma}}+\sum_{\beta=1}^{\infty}\left(1-e^{2^{-\beta}}\right) \\
& \approx \log _{2} n-0.521865+0.854613=\log _{2} n+0.332747
\end{aligned}
$$

Thus, for sufficiently large values of $n$,

$$
E_{n}(X) \approx \log _{2} n+0.332747 .
$$


Given Theorem 11, the key remaining challenge is thus to efficiently and securely find a closest node identifier (with distance measured in terms of leading overlapping bits) to a random key in an unstructured network.

In our design, all nodes in the network periodically participate in a global network size estimation operation at a frequency of $f$. Each round results in all peers learning a discrete approximation $p$ (the number of overlapping leading bits for a particular random key) for $\bar{p}$ (the theoretically expected number of overlapping leading bits). The specific frequency $f$ is chosen based on the expected level of network churn and the desired accuracy. $f$ is a design parameter and fixed in the implementation. The results from the last $k$ iterations are averaged locally by each peer to obtain an approximation $\tilde{p}$ for $\bar{p}$. A standard deviation can also be computed if an estimate for the error of the size estimate is desired. Furthermore, the current $p$ value is used by the protocol as a parameter to (slightly) improve the performance for the next round. We will refer to the number of overlapping leading bits from the previous round as $p^{\prime}$.

\subsection{Generating a Random "Key"}

Given a frequency $f$, the random target key $T$ for each round is generated by hashing the start time $S$, which is the absolute UTC time at times that are zero modulo $f$. For example, if $f=1 h$, then a fresh key could be generated every hour by hashing "DD-MM-YYYY HH:00:00". Using this method, all peers will generate exactly the same key at (roughly) the same time. Generating the key this way has the advantage that it will be known to all peers without communication and that malicious participants cannot influence the process. However, it should be noted that while the keys satisfy the statistical properties of being random and uniform, it is trivial to compute them in advance.

Our method requires all peers to calculate the current key $T$ at the respective start time $S$. The network size estimation protocol's goal is to communicate to all peers an identity $I_{T}$ of a peer with the largest proximity $p$ with respect to $T$. More specifically, all peers are supposed to learn one of the closest peer identities $I_{T}$ between time $S$ and time $S+f$. Given $I_{T}$, each peer can then calculate $p$, the average $\tilde{p}$ of the $p$-values from the last $k$ rounds and finally the current network size estimate $2^{\tilde{p}-0.332747}$.

Note that $p$ is a discrete value representing the number of leading matching bits between the key $T$ and a peer's identity. As such, it is quite likely that many peers have identities with the same number of leading matching bits and hence the same proximity $p$. Our protocol deliberately ignores all bits after the first mismatch to improve performance; if multiple peers have the same proximity score, it does not matter which of these equivalent identities is propagated as they will all ultimately result in the same proximity estimate $p$.

\subsection{Starting the Flood}

Our protocol essentially floods the network with the identity of a closest peer $I_{T}$. If only the identities of closest peers are propagated, this operation would create 
less than $2|E|$ messages (up to two per edge in the network). The challenge is to avoid creating significantly more than $2|E|$ messages, which is difficult since in an unstructured network a peer with the closest identity $I_{T}$ cannot be certain that there is no other peer that is closer to $T$. We address this problem by delaying the flood based on proximity.

First, each peer evaluates its own proximity $x$ with respect to $T$. How close the peer is to $T$ is then used to determine how soon the peer will initiate the flood of the network with a message claiming that he is the closest peer. We use the previous network size estimate as a guide to time the release. Specifically, given a proximity of $x$ leading overlapping bits and a network size estimate $p^{\prime}$ from the previous round, we use the following function to determine the time when a peer starts to flood the network:

$$
r(x):=S+\frac{f}{2}-\frac{f}{\pi} \cdot \arctan \left(x-p^{\prime}\right)
$$

Using this function, if the peers proximity $x$ is equal to the proximity of the last iteration, the peer floods the network at time $S+\frac{f}{2}$. If the peer's proximity is 0 bits, the peer floods the network close to time $S+f$, which is at the end of the time interval for the current round; if the peer's proximity is significantly higher than $p^{\prime}$, the peer floods at the beginning of the round, which is close to time $S$. It should be noted that since $\lim _{x \rightarrow p^{\prime}} \frac{\partial r}{\partial x}(x)=1$, Equation 5 maximizes the difference between release times for nodes with proximities that are close to the previous number of overlapping bits $p^{\prime}$ and as such minimizes the chance of two peers releasing floods for different network size estimates around the same time - assuming the network size estimate did not change significantly.

\subsection{Processing the Flood}

Peers that receive the resulting network size estimation messages first perform a series of validation steps before continuing to forward the message. First, each peer checks if a notification from a closer peer has already been received for round $S$. Messages with proximity scores equal to the currently known best score for the current round are simply discarded. Messages with lower proximity scores should only occur if there is significant clock skew, and are answered immediately with a message indicating the higher proximity score. If the message contains a higher proximity than what was previously known for the current round, the peer checks if the proximity $p$ of the given message justifies receiving it at the current time. If not, further processing is delayed until the local peer's time is past $r(p)$. Finally, before forwarding and further processing, the format of the message is validated (this is discussed in more detail in Section 3.5).

Assuming the message validates, the peer then proceeds to forward it to all of its neighbors. For each neighbour, the message is forwarded with a peer-specific random delay. If a peer receives a message with an equivalent proximity score during the delay, the transmission is canceled. As a consequence, the delay helps to both avoid an explosion of messages on the network in a tiny amount of time, and to improve the chances of traversing each edge in only one direction per link 
(as it decreases the chances of equivalent messages being sent in both directions at the same time).

The permissible delay $L$ is calculated using the time difference between $r(p)$ and $r(p-1)$ divided by an estimate of the network's diameter. The network diameter $D$ is estimated using the maximum of the hop counters in the network size estimation messages from the previous $k$ iterations. For each neighbor, the peer then applies a delay chosen uniformly at random from the interval $[0, L)$ where $L$ is defined as

$$
L:=\frac{r(p-1)-r(p)}{D} .
$$

As a result, each peer in the network is expected to receive a proximity notification with proximity $p$ before any peer with notifications for proximity $p-1$ would even begin to flood the network. Naturally, there are various causes that could increase the number of messages above $|E|$ in a real-world network; for example, different system times between peers, high network latencies, and peers with unusually high distances to $I_{T}$ can increase the total number of messages. However, all benign peers that form a connected component are guaranteed to eventually receive $I_{T}$. Furthermore, given that the number of bits in the key is a small constant, the total number of messages can never exceed $O(|E|)$ per round.

\subsection{Joining the Network}

A peer that is freshly joining the network lacks results from previous rounds for network size estimation. In order to bootstrap the protocol, each peer starts with a network size estimate based on its own key in relation to the key $T$ from the previous round, and a network diameter estimate of one. Whenever a connection between two peers is established, they exchange the network size estimation result from the previous round (and the current round if their local time is past $f(p))$. As a result, all nodes can always be expected to use the same value for $p^{\prime}$ in Equation (5).

\subsection{Proof of Work}

The presented design is vulnerable to an adversary that creates fake identities (Sybil attack [2]). Such an adversary could create identities that are "close" to the respective key for each time $S+\mathbb{Z} r$. By flooding the network with the respective messages at the right time, the adversary can then make the network appear to be larger than it is.

Our design defends against this attack by requiring a proof of work [19] for the identity of the peer as part of the network size estimation message. Specifically, we require the originator to produce a value with a $W$-bit hash collision with the peer's identifier, and a cryptographic signature to demonstrate that the identifier was derived from a valid public-private key pair.

The complete message format for the network size estimation messages is described in our technical report [7]. 


\section{Security Analysis}

For our security analysis, we assume that an active adversary is participating in the $\mathrm{P} 2 \mathrm{P}$ network. The adversary is allowed to control a certain percentage of colluding malicious nodes in the network. Individual malicious and benign nodes are assumed to have the same amount of computational resources; all nodes are assumed to have sufficient bandwidth to participate in the protocol in the absence of an attack.

We can imagine three different high-level goals an adversary may pursue with an attack. First, an adversary may try to cause nodes to significantly underestimate the size of the network. Second, an adversary may try to cause nodes to significantly overestimate the size of the network. Finally, an adversary may want to use the protocol for a denial-of-service attack where the $\mathrm{P} 2 \mathrm{P}$ network uses significantly more traffic for network size estimation, possibly causing other components of the system to be left with insufficient bandwidth.

The best method for an adversary to cause peers to underestimate the size of the network is to not participate in the protocol. If the adversary controls $X \%$ of the network, that will cause the protocol to underestimate the size of the network by $X \%$. Furthermore, if the adversary is able to control an $\epsilon$-separator of the network graph 91013] (removing an $\epsilon$-separator from a graph reduces the size of the largest remaining connected component to $\epsilon n$ ), then the overall network size estimate would be reduced to less than $\epsilon n$ for all nodes in the network. Given that in all of these cases the network size estimate would correspond to the size of the network after the removal of the adversaries' nodes, this attack is not particularly disruptive in relation to the strength of the adversary. Thus, an adversary cannot make the network appear significantly smaller than it is.

If the adversary wants to make the network look larger by $M$ nodes, it needs to first compute (and store) $M$ public-private key pairs. Then, at every time interval $f$, the adversary needs to compute collisions costing an additional $O\left(2^{W}\right)$ to generate the required $W$-bit collision. Actually joining the network with $M$ "fake" peers is not required. If $W$ is chosen so large that the adversary cannot solve the problem at frequency $f$, it is still possible for the adversary to cause an increase in the network size estimate by solving the problem every $c \cdot f$ (for an appropriate choice of $c$ based on the adversaries computational resources), which would still affect the computed medium-term averages computed for subsequent intervals. Using such an attack, an adversary can make the network appear significantly larger than it is, as long as the adversary has access to sufficient computational resources.

Finally, for a denial-of-service attack, an adversary would first generate additional identities and generally perform the same steps as for increasing the estimated network size. Now, suppose the adversary has created $m$ identities that are closer to the current key than the closest actual peer in the network by $1 \ldots m$-bits respectively. Then, just after the identity of the peer that is actually closest to the key has been broadcast to the network, the adversary can cause $m$ additional broadcasts by transmitting its $m$ "fake" identities in order of increasing proximity to the key. Each time, the network will presume that 
a closer peer was "late" with its transmission (for example, due to clock-skew or network latency) and broadcast the update. If the network is already of size $n$, the expected one-time cost for the adversary to create $m$ such identities is $O\left(n 2^{m}\right)$; the attack then requires an additional $O\left(m 2^{W}\right)$ operations for the hash collisions at frequency $f$. Therefore, if we neglect the high one-time cost of computing identities, the adversary can cause $|E|$ traffic on the network at the cost of $O\left(2^{W}\right)$ computations.

\section{Analytical Worst-Case Analysis}

The following scenario describes the theoretical worst case in terms of bandwidth consumption by the protocol. Without loss of generality, suppose a 512-bit hash function is being used. Then, the worst-case network would for $\mu \in[1,512]$ have exactly one peer with $\mu$ matching bits with the target key $T$ (in each round); all other peers in the network would have zero matching bits. The peers that do have matching bits should be connected to the main network via a long chain (with larger distances for peers with larger $\mu$ ), causing the network diameter $D$ to be large. (As a result, the algorithm will calculate $L \approx 0$.) Peers with $\mu$ matching bits should furthermore have (or pretend to have) a late system time that causes them to transmit effectively at time $S+\mu \epsilon$; all other peers have fast clocks that cause them to accept any message at any time, causing 512 network-wide floods per round. Furthermore, in the worst case, network delays in the main network would be so large that each of the 512 messages would traverse each link in both

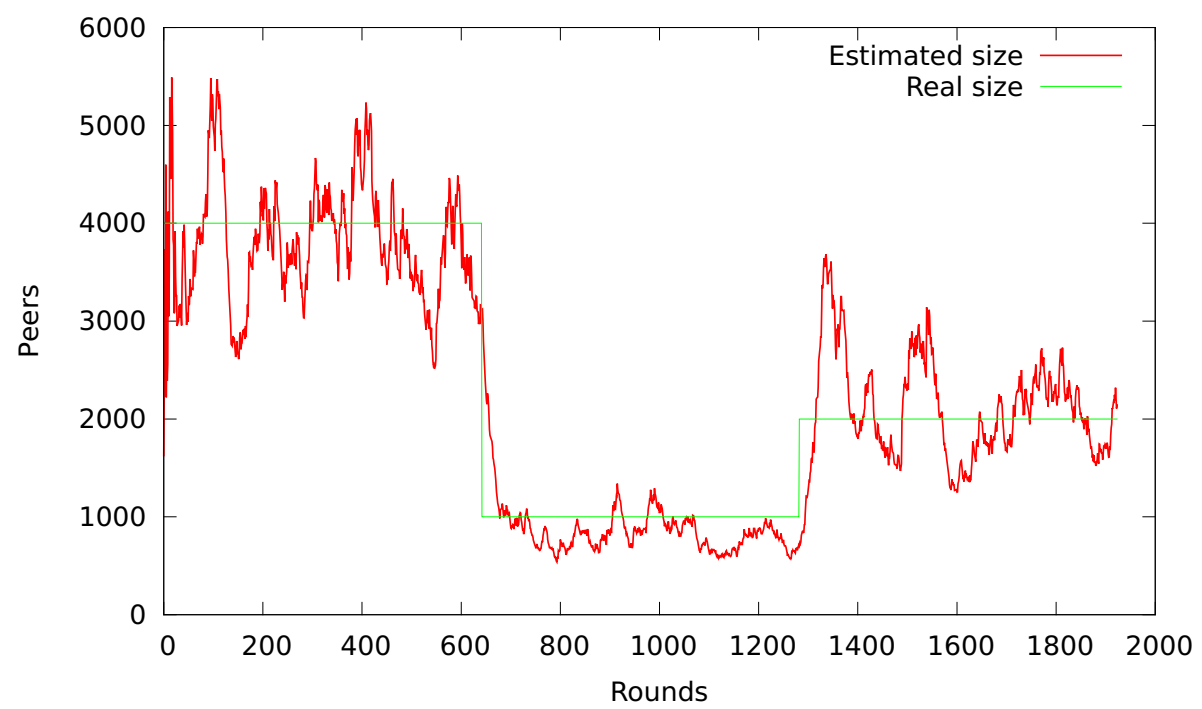

Fig. 1. Network size estimates as actually observed by a node in relation to the (changing) total network size over time for a random graph topology. All nodes arrive at the same estimate, except for nodes that recently joined the network. 
directions, creating a total of $1024 \cdot|E| \in O(|E|)$ transmissions. Note that this scenario covers the worst-case and includes an adversary with infinite computing power and full control over the network topology.

\section{Experimental Results}

We have implemented the presented protocol in the GNUnet P2P framework 1 , and evaluated the behavior of the proposed protocol using large-scale emulation [5].

To evaluate the network size estimation quality, we show the network size estimate based on the average of the previous 64 rounds. Figure 1 shows the evolution of the network size estimate for a random graph topology [35] with a minimum node degree of 5 and an average node degree of 10 for the 4,000 node network. It should be noted that the shape of the network topology has no impact on the size estimate. The experiment was started with an initial network size of 4,000 nodes for 640 rounds. Then, we decreased the network size to 1,000 nodes for 640 rounds (the remaining peers stayed connected) and finally increased it to 2,000 nodes for another 640 rounds.

The number of rounds used to calculate the result has an impact on the precision of the estimate. The trade-off between more measurements and the resulting precision is plotted in Figure 2. Precision is measured as $|\tilde{p}-\bar{p}|$. Averaging over

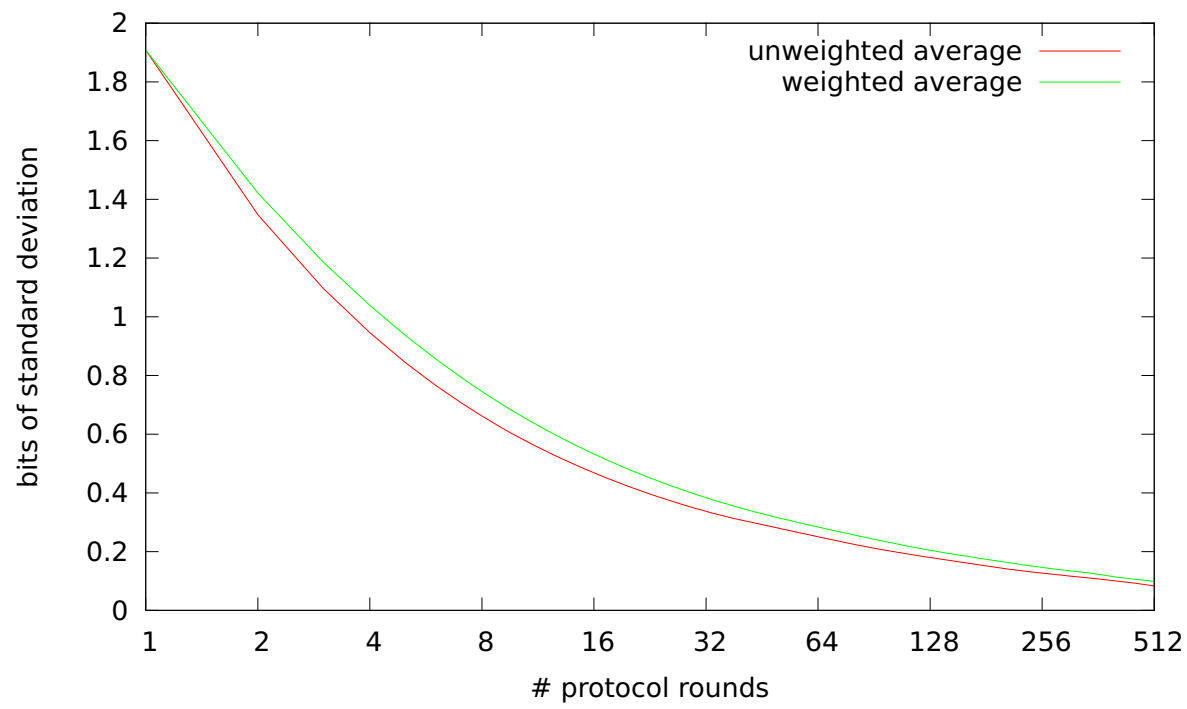

Fig. 2. Trade-off between the precision of the network size estimate vs the number of rounds used to calculate $\tilde{p}$. Naturally, this plot assumes that the network size does not change during the measurement.

${ }^{1}$ https://gnunet.org/svn/gnunet/src/nse/ 
four rounds gives results with a standard deviation of one. As the network size is calculated as $2^{\bar{p}-0.332747}$ (Theorem 11), a standard deviation of one means that the network size estimate is in an interval between half and double the actual network size $68 \%$ of the time and between a quarter and four times the actual network size $95 \%$ of the time. The 64 rounds we used for Figure 1 correspond to a standard deviation of under 0.3 . This means that $95 \%$ of the time the network size estimate is accurate up to a factor of $\approx 1.5$.

In the proof for Theorem 1 we made an approximation that is valid if " $n$ is sufficiently large". However, what constitutes a sufficiently large $n$ in practice is not obvious. Figure 3 shows the results of a simulation that determined $\tilde{p}$ from 50,000 rounds for networks of size $n \in\left[1,2^{24}\right]$. The difference $\tilde{p}-\log _{2} n$ quickly converges to the constant calculated in Theorem 1 (0.332747). It should be noted that even with 50,000 rounds the values for $\tilde{p}$ still exhibit some visible fluctuation. Figure 3 shows that for a reasonable number of rounds of measurement $(\leq$ $50,000)$, the "sufficiently large values of $n$ " are values larger than $2^{5}$.

Additional experimental results, such as an analysis of the impact of the network topology and clock skew on bandwidth consumption, can be found in our technical report [7. The key result from those experiments is that the protocol

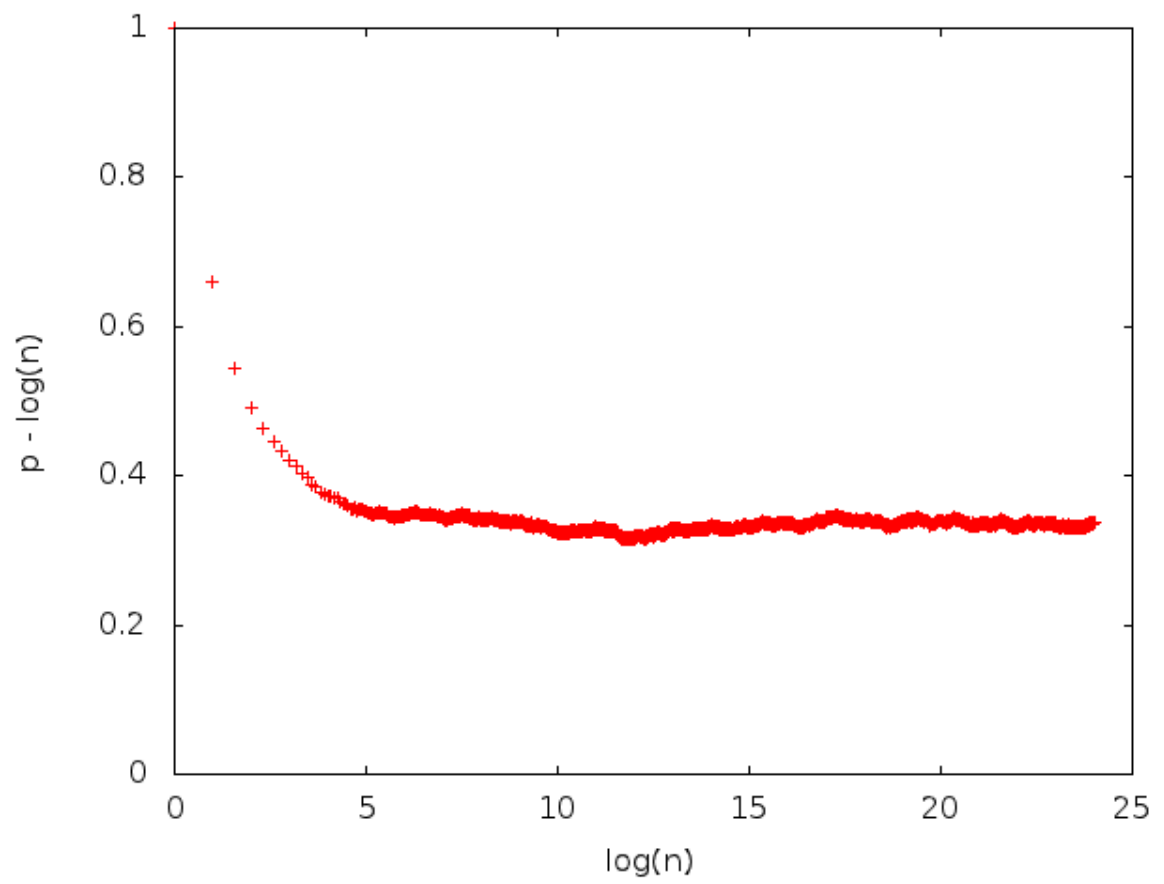

Fig. 3. Differences observed between $\log _{2} n$ and the average observed value for $p$ over 50,000 iterations in relation to the network size. The average difference was $0.33 \approx 1 / 3$. Since peer-to-peer networks smaller than $2^{5}=32$ peers are not really relevant for network size estimation techniques, we use a uniform correction of $1 / 3$ to compensate for the observed difference when estimating $n$ from $\bar{p}$. 
works for any topology, tolerates clock skew and under realistic conditions uses fewer than $2|E|$ messages per round.

\section{Conclusion}

We have presented the first protocol for securely and efficiently estimating the size of a $\mathrm{P} 2 \mathrm{P}$ network. Our protocol combines proximity to a deterministic sequence of (pseudo-)random values, staggered triggering of messages and a proof-of-work component. The scheme works for structured and unstructured networks, is inexpensive in terms of bandwidth, perfectly distributed imposing equal requirements in terms of computation and bandwidth on all nodes, and is quite accurate even for networks under churn. The protocol is secure against adversaries trying to make the network appear smaller and makes it computationally expensive (based on a parameter $W$ ) to make the network appear larger or to flood the network with unwarranted traffic.

Acknowledgments. This work was funded by the Deutsche Forschungsgemeinschaft (DFG) under ENP GR 3688/1-1. We thank Mikhail Atallah for his help proving Theorem 11 and Christopher Wolf for an insightful discussion on an earlier draft of this paper.

\section{References}

1. Bustos-Jimenez, J., Bersano, N., Schaeffer, S.E., Piquer, J.M., Iosup, A., Ciuffoletti, A.: Estimating the size of peer-to-peer networks using lambert's w function. In: Gorlatch, S., Fragopoulou, P., Priol, T. (eds.) Grid Computing, pp. 61-72. Springer, US (2008)

2. Douceur, J.R.: The Sybil Attack. In: Druschel, P., Kaashoek, M.F., Rowstron, A. (eds.) IPTPS 2002. LNCS, vol. 2429, pp. 251-260. Springer, Heidelberg (2002)

3. Erdős, P., Rényi, A.: On random graphs. I. Publ. Math. Debrecen 6, 290-297 (1959)

4. Eugster, P.T., Guerraoui, R., Handurukande, S.B., Kouznetsov, P., Kermarrec, A.-M.: Lightweight probabilistic broadcast. ACM Trans. Comput. Syst. 21, 341$374(2003)$

5. Evans, N., Grothoff, C.: Beyond simulation: Large-scale distributed emulation of p2p protocols. In: 4th Workshop on Cyber Security Experimentation and Test (CSET 2011). USENIX Association (2011)

6. Evans, N., Grothoff, C.: R5n: Randomized recursive routing for restricted-route networks. In: 5th International Conference on Network and System Security. IEEE, Milan (2011)

7. Evans, N., Grothoff, C., Polot, B.: Efficient and secure decentralized network size estimation. Technical report, Technische Universität München (2011)

8. Jelasity, M., Montresor, A., Babaoglu, O.: Gossip-based aggregation in large dynamic networks. ACM Trans. Comput. Syst. 23, 219-252 (2005)

9. Kernighan, B.W., Lin, S.: An Efficient Heuristic Procedure for Partitioning Graphs. The Bell System Technical Journal 49(1), 291-307 (1970)

10. Kleinberg, J., Sandler, M., Slivkins, A.: Network failure detection and graph connectivity. SIAM J. Comput. 38, 1330-1346 (2008) 
11. Kostoulas, D., Psaltoulis, D., Gupta, I., Birman, K., Demers, A.: Decentralized schemes for size estimation in large and dynamic groups. In: Proceedings of the Fourth IEEE International Symposium on Network Computing and Applications, pp. 41-48. IEEE Computer Society, Washington, DC (2005)

12. Malkhi, D., Naor, M., Ratajczak, D.: Viceroy: a scalable and dynamic emulation of the butterfly. In: PODC 2002: Proceedings of the Twenty-First Annual Symposium on Principles of Distributed Computing, pp. 183-192. ACM, New York (2002)

13. Marx, D.: Parameterized graph separation problems. Theor. Comput. Sci. 351, 394-406 (2006)

14. Massoulié, L., Le Merrer, E., Kermarrec, A.-M., Ganesh, A.: Peer counting and sampling in overlay networks: random walk methods. In: Proceedings of the Twenty-Fifth Annual ACM Symposium on Principles of Distributed Computing, PODC 2006, pp. 123-132. ACM, New York (2006)

15. Maymounkov, P., Mazières, D.: Kademlia: A Peer-to-Peer Information System Based on the XOR Metric. In: Druschel, P., Kaashoek, M.F., Rowstron, A. (eds.) IPTPS 2002. LNCS, vol. 2429, pp. 53-65. Springer, Heidelberg (2002)

16. Le Merrer, E., Kermarrec, A.-M., Massouli, L.: Peer to peer size estimation in large and dynamic networks: A comparative study. In: 15th IEEE International Symposium on High Performance Distributed Computing 2006, pp. 7-17 (2006)

17. Polot, B.: Adapting blackhat approaches to increase the resilience of whitehat application scenarios. Master's thesis, Technische Universität München (2010)

18. Rowstron, A., Druschel, P.: Pastry: Scalable, Decentralized Object Location, and Routing for Large-Scale Peer-to-Peer Systems. In: Guerraoui, R. (ed.) Middleware 2001. LNCS, vol. 2218, pp. 329-350. Springer, Heidelberg (2001)

19. Serjantov, A., Lewis, S.: Puzzles in p2p systems. In: 8th CaberNet Radicals Workshop, Corsica (2003)

20. Shafaat, T.M., Ghodsi, A., Haridi, S.: A Practical Approach to Network Size Estimation for Structured Overlays. In: Hummel, K.A., Sterbenz, J.P.G. (eds.) IWSOS 2008. LNCS, vol. 5343, pp. 71-83. Springer, Heidelberg (2008) 